\title{
Design of Measurement Algorithm of Feedback Strength Factor of Optical Feedback Self-mixing Interferometry Systems based on System Generator
}

\author{
Wen Zhao ${ }^{1}$, Huiying $\mathrm{Ye}^{2}$ \\ School of Information Engineering \\ Zhengzhou University \\ Zhengzhou, China \\ zzuzhaowen@163.com
}

\author{
Yanguang $\mathrm{Yu}^{3}$ \\ School of Electrical Computer and \\ Telecommunications Engineering \\ University of Wollongong \\ Wollongong, Australia
}

\begin{abstract}
The spectrum features of the external light phase of a semiconductor laser without optical feedback and with optical feedback are analysed based on the Lang-Kobayashi equations, which contributes to putting forward the measurement algorithm of feedback strength factor of optical feedback self-mixing interferometry systems in the frequency domain. The hardware models of this algorithm are established by applying XILINX System Generator tools on the Matlab/Simulink software platform. Finally, the associated simulations for verification of the measurement algorithm in different feedback levels are carried out and it is proved effectively.
\end{abstract}

Keywords-measurement and metrology; optical feedback self-mixing interferometry; feedback strength factor ; System Generator

\section{INTRODUCTION}

Optical feedback self-mixing interferometry (OFSMI) effect refers to that in laser application system, when an external target exists, the original light emitted by a semiconductor laser (SL) will be mixed with part of the light refected or scattered by the external target in the resonant cavity of the SL, after which the power as well as the frequency of the SL will be modulated. As the feedback light carries motion and status information of the external reflective targets, so it can be applied to measure the parameters of the moving targets and the parameters of the SL itself, such as the measurement of displacement[1], vibration[2], velocity[3], the linewidth enhancement factor of the SL[4] and so on. The feedback strength factor (denoted by C) is an important parameter of optical feedback self-mixing interferometry systems, which reflects the feedback level of the system and affects the laser's intensity, the optical noise, the spectrum effect, the linewidth enhancement etc. According to the different $C$ values, the OFSMI effect can be divided into three situations: weak feedback, moderate feedback and strong feedback. A measure model of $C$ factor is established based on Lang-Kobayashi equations and a measurement algorithm of $C$ factor in the frequency domain is proposed in this paper. And then, we apply XILINX System Generator, a kind of system design tool for DSP, to establish the system-level hardware models and simulate effectively and quickly due to the parameterized modules and the graphical design of the System Generator.

\section{THE DESIGN OF THE MEASUREMENT ALGORITHM OF $C$ IN FREQUENCY DOMAIN}

The general measure model based on the OFSMI theory has been raised as follows [5-7]:

$$
\begin{gathered}
\phi_{F}(t)=\phi_{0}(t)-C \cdot \sin \left[\phi_{F}(t)+\arctan (\alpha)\right] \\
g(t)=\cos \left(\phi_{F}(t)\right) \\
p(t)=p_{0}[1+m \cdot g(t)]
\end{gathered}
$$

Equation (1) describes the phase variation due to the optical feedback, where $o(t)$ and ${ }_{F}(t)$ are the external light phases of a SL without and with optical feedback respectively. Equation (2) is called interferometric function. $g(t)$ is called OFSMI signal which represents the modulation level of external cavity's light phase acts on the output power of the SL. Equation (3) gives the output power of the $\mathrm{SL}$, denoted by $p(t)$, where $\mathrm{p}_{0}$ is the power emitted by the free running SL, and $\mathrm{m}$ is modulation index which typical value is about $10^{-3}$.

Taking Fourier transform, denoted by $F\{\}$, on both sides of equation (1), we have

$$
\phi_{F}(f)=\phi_{0}(f)-C \cdot F\left\{\sin \left[\phi_{F}(t)+\arctan (\alpha)\right]\right\}
$$

Where $F(f)$ and $o(f)$ are the Fourier transforms of

${ }_{F}(t)$ and $o(t)$ respectively. For ease of manipulation, we can use a function

$$
\phi_{1}(t)=\sin \left[\phi_{F}(t)+\arctan (\alpha)\right]
$$

So that equation (4) becomes:

$$
\phi_{F}(f)=\phi_{0}(f)-C \cdot F\left\{\phi_{1}(t)\right\}=\phi_{0}(f)-C \cdot \phi_{1}(f)
$$

Where ${ }_{l}(f)$ is the Fourier transform of ${ }_{l}(t)$.

The output power of the SL is $p(t)=p_{0}[1+m \cdot g(t)]$, and after normalizing it, we can get the OFSMI signal $g(t)$. Then ${ }_{F}(t)$ can be figured up by calculating the inverse function of $g(\mathrm{t})$ according to equation (2). Thereafter, we calculate the spectrum of $F(t)$, that is $F(f)$. Meanwhile, we are able to work out $\quad l(t)$ using equation (5) and calculate its spectrum ${ }_{l}(f)$.

If the external target moves in a manner close to simple harmonic vibration, ${ }_{0}(t)$ will be close to a sinusoidal, in which case it can be considered as of narrow band in frequency domain. From equations (1) and (4), $\quad F(t)$ should exhibit a broader spectrum than on $o(t)$ because of the nonlinear mapping from $\quad 0(t)$ tọ ${ }_{F}(t)$. In other wordș, ${ }_{F}(f)$ and ${ }_{l}(f)$ should spread over a wider frequency range than $o(f)$ does. Now, we can divide the frequency range of 
non-vanishing spectrum of $\quad F(f)$ and ${ }_{l}(f)$ into two domains, denoted by ..1 and respectively, in which the spectrum of $\sim(f)$ is non-vanishing or vanishing, i.e.:

$$
\begin{gathered}
.{ }_{1}: \quad f \in\left\{\phi_{F}(f) \neq 0, \phi_{1}(f) \neq 0, \phi_{0}(f) \neq 0\right\} \text { and } \\
: \quad f \in\left\{\phi_{F}(f) \neq 0, \phi_{1}(f) \neq 0, \phi_{0}(f)=0\right\} .
\end{gathered}
$$

In the frequency range of .equation (6) equals to:

$$
\phi_{F}(f)=-C \cdot \phi_{1}(f), f \in \Omega_{2}
$$

herefore $\mathrm{C}$ can be calculated as:

$$
C=\frac{\left|\phi_{F}(f)\right|}{\left|\phi_{1}(f)\right|}, f \in \Omega_{2}
$$

The above relation is valid for all the frequency components in .. In practice, we can employ a summation over all frequency components in . to increase the accuracy of the estimate:

$$
C=\frac{\sum_{f \in \Omega_{2}}\left|\phi_{F}(f)\right|}{\sum_{f \in \Omega_{2}}\left|\phi_{1}(f)\right|}
$$

To apply the method, we shall identify . and .. In the cases of harmonic vibration, $\quad o(t)$ is a periodic function with a fundamental frequency $f_{0}$. Then $o(f)$ should exhibit a large fundamental component at $f_{0}$, and some harmonic components. The highest harmonic component will in general depend on the actual waveform, and in practice we consider $15 f_{0}$ as the upper frequency necessary to cover well the spectrum details. In terms of the upper bound of which is denoted by $f_{M}$ we simply choose $f_{M}$ as high as possible while observing $\phi_{F}(f) \neq 0$, and $\phi_{1}(f) \neq 0$. In this paper we have taken . to be $15 f_{0}, f_{M}$

On the basis of the theory above, the measurement algorithm is designed as follows:

- $\quad$ Step 1. Normalize the signal $p(t)$ to get $g(t)$

- Step 2. Carry out inverse cosine function operation on $g(t)$ and then phase-unwrapping to obtain $\quad F(t)$.

- Step 3. Calculate ${ }_{l}(t)$ using equation (5).

- $\quad$ Step 4. Apply DFT on ${ }_{F}(t)$ and ${ }_{l}(t)$ and obtain their spectrums.

- $\quad$ Step 5. Calculate $C$ value using equation (9).

\section{ESTABLISH THE HARDWARE MODELS BASED ON SYSTEM GENERATOR}

XILINX System Generator is a modeling design platform which is specially applied for DSP. It combines with the powerful operation circumstance that Matlab/Simulink offered to conduct the graphical design and some simulations, establish the algorithm model, and then produce the corresponding hardware project files.

When to build the hardware models for the measurement algorithm of $C$ factor by using System Generator, they mainly include two important parts: the unwrapping module of the external light phase and the spectrum analysis module of the external light phase.

Build the unwrapping model of the external light phase as shown in Fig. 1.It has used the ROM module to carry out the inverse trigonometric function by applying the lookup table method.

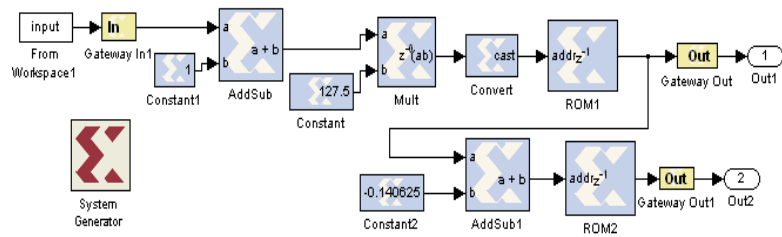

Figure 1. Unwrapping module of the external light phase

Build the spectrum analysis model of the external light phase as shown in Fig. 2. The crucial parts in this module are the FFT module and the design for complex multiplication. We can either self-design the FFT module, or use the existing IP core, and we choose the latter, that is using the FFT IP core embedded in the DSP modules of System Generator.

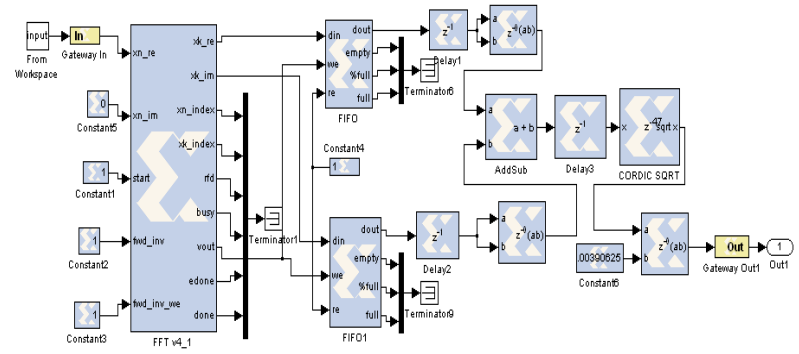

Figure 2. Spectrum analysis module of the external light phase

\section{SIMULATIONS AND ANALYSIS}

In this paper, we preset $C=5, \alpha=3$ to get OFSMI signal $g(n)$ according to Eqs.(1),(2),(3), and import the data to the hardware models presented in section III above to verify the $C$ value solved by the modules whether equals to the preset $C$ value.

As is showed in Fig. 3, when $f>3000 \mathrm{~Hz}, \quad{ }_{0}(f)=0$ but $\phi_{1}(f) \neq 0$. Hence the lower band of $\Omega_{2}$ can be set as $3000 \mathrm{~Hz}$, while the upper band of it can be set as $12000 \mathrm{~Hz}$. By looking at the spectrum at $f=4800 \mathrm{~Hz} \in \Omega_{2}$, we $\operatorname{get} \varphi_{F}(4800)=0.5127, \varphi_{1}(4800)=0.1025$, and put these data into Eq.(8): $C=0.5127 / 0.1025=5.0020$. It can be calculated that the relative error between the $\mathrm{C}$ value solved by the modules and the preset $\mathrm{C}$ value is $0.04 \%$. Set the range of $\Omega_{2}: f \in[3000 \mathrm{~Hz}, 12000 \mathrm{~Hz}]$, and apply Eq.(9) to calculate $\mathrm{C}=5.0010$, and the relative error is $0.02 \%$.

Extensive simulations have been done for verification of the hardware models proposed in section III. We preset some different $\mathrm{C}$ values covering a range of the three levels of optical feedback and different $\alpha$ values, and get the calculated $\mathrm{C}$ values as the following table shows. 

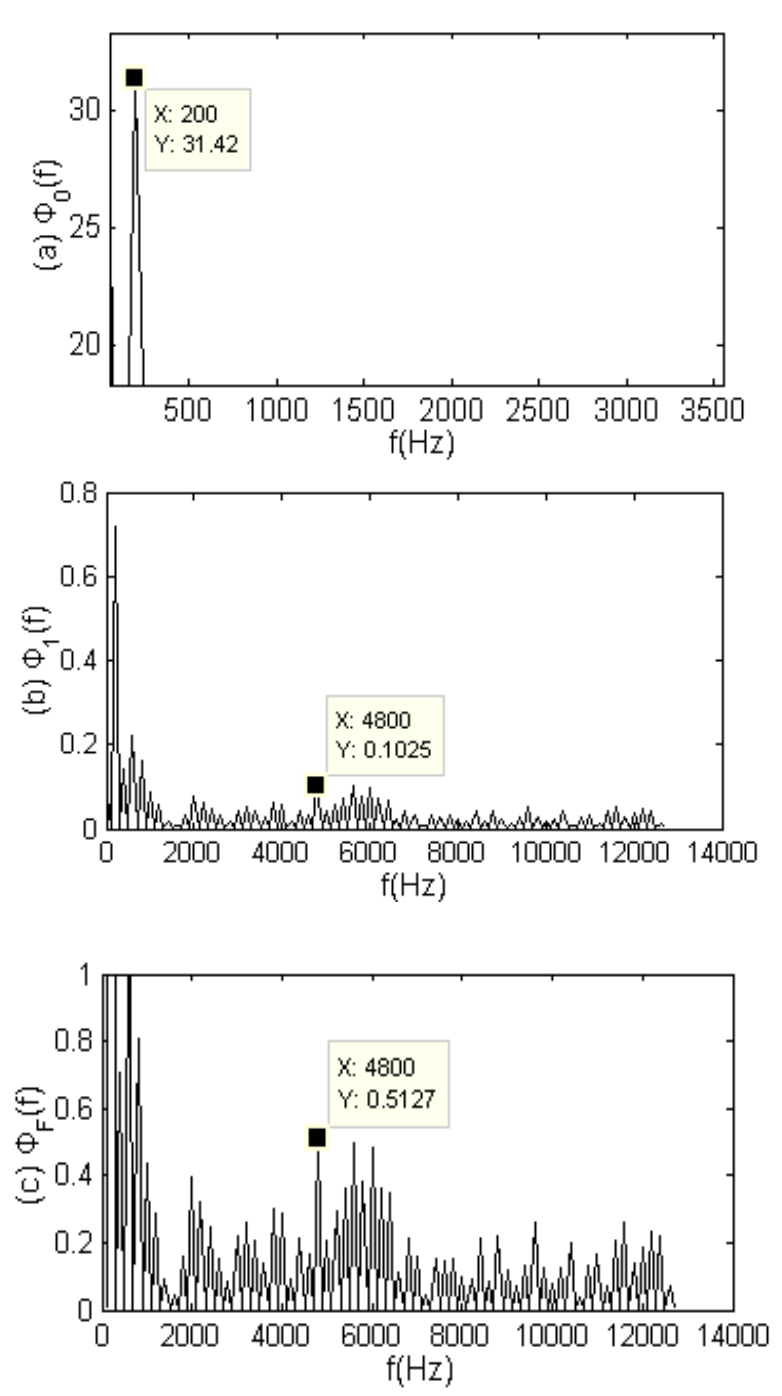

Figure 3. $\begin{array}{lllll}\text { (a) spectrum of } \quad{ }_{0}(t) & \text { (b) spectrum of } \quad{ }_{l}(t) \quad \text { (c) spectrum }\end{array}$ of ${ }_{F}(t)$

TABLE I. CALCULATED $C$ VALUES USING PROPOSED METHOD

\begin{tabular}{|c|c|c|c|c|}
\hline$\alpha$ & $\alpha=1$ & & $\alpha=3$ & \\
\hline $\begin{array}{c}\text { Preset } \\
C\end{array}$ & $\begin{array}{c}\text { Calculated } \\
C\end{array}$ & $\begin{array}{c}\text { Relative } \\
\text { Error (\%) }\end{array}$ & $\begin{array}{c}\text { Calculated } \\
C\end{array}$ & $\begin{array}{c}\text { Relative } \\
\text { Error (\%) }\end{array}$ \\
\hline 1 & 0.9999 & 0.010 & 0.9994 & 0.060 \\
\hline 1.5 & 1.5005 & 0.033 & 1.5001 & 0.007 \\
\hline 2 & 2 & 0 & 2 & 0 \\
\hline 2.5 & 2.5 & 0 & 2.5 & 0 \\
\hline 3 & 3 & 0 & 3 & 0 \\
\hline 3.5 & 3.5014 & 0.040 & 3.5012 & 0.034 \\
\hline 4 & 4 & 0 & 4 & 0 \\
\hline 4.5 & 4.4987 & 0.029 & 4.4990 & 0.028 \\
\hline 5 & 5.0020 & 0.040 & 5.0010 & 0.020 \\
\hline
\end{tabular}

\begin{tabular}{|l|l|l|l|l|}
\hline 10 & 10.0043 & 0.043 & 10.0043 & 0.043 \\
\hline \multicolumn{5}{|c}{} \\
\hline
\end{tabular}

\section{CONCLUSION}

This paper presents a measurement algorithm of feedback strength factor of optical feedback self-mixing interferometry systems in the frequency domain, and applies System Generator to finish the hardware models design of this algorithm. Compared with existing measurement algorithm based on the time domain, the algorithm presented in this paper covers a large range of $C$ values. Effectiveness of the proposed method has been confirmed by the simulations.

\section{REFERENCES}

[1] M. Norgia, S. Donati, "A displacement-measuring instrument utilizing self-mixing interferometry,"J. IEEE Transactions on Instrumentation and Measurement, vol.52, pp.1765-1770, June 2003.

[2] Franck Chollet, Gopal M.Hegde, Zhang Xuming, et al. "Vibration measurement with a micromachined mirror in a very-short external cavity laser," J. Sensors and Actuators. Vol.116, pp.232-240, 2004.

[3] Xavier Raoul, Thierry Bosch, G.uy Plantier and Noel Servagent, "A double laser diode on board sensor for velocity measurements," J. IEEE Transactions on Instrumentation and Measurement, vol.53, pp.95-101, 2004.

[4] Jiangtao Xi, Yanguang Yu, Joe F.Chicharo, et al. "Estimating the parameters of semiconductor lasers based on weak optical feedback self-mixing interferometry," J. IEEE Quantum Electronics, vol.41, pp.1058-1064, August 2005.

[5] Yanguang Yu, Guido Giuliani, and Silvano Donati, "Measurement of the linewidth enhancement factor of semiconductor lasers based on the optical feedback self-mixing effect ," J. IEEE Photonics Technology Letters, vol.16, pp.990-992, April 2004.

[6] Yanguang Yu, Jiangtao Xi, J.F.Chicharo, and T.Bosch, "Toward Automatic Measurement of the Linewidth-Enhancement Factor Using Optical Feedback Self-Mixing Interferometry, "IEEE Journal of Quantum Electronic, vol.43, pp.527-534,July 2007.

[7] Lu Wei, Jiangtao Xi, Yanguang Yu, Joe Chicharo, "Phase Unwrapping of Self-mixing Signals Observed in Optical Feedback Interference for Displacement Measurement," IEEE ISPACS, pp.780-783,2006. 